\title{
On a Subclass of Meromorphic Close-to-Convex Functions
}

\author{
Ming-Liang Li, ${ }^{1,2}$ Lei Shi, ${ }^{3}$ and Zhi-Gang Wang ${ }^{3}$ \\ ${ }^{1}$ School of Mathematics and Statistics, Central South University, Changsha, Hunan 410075, China \\ ${ }^{2}$ School of Mathematics and Computing Science, Hunan First Normal University, Changsha, Hunan 410205, China \\ ${ }^{3}$ School of Mathematics and Statistics, Anyang Normal University, Anyang, Henan 455000, China \\ Correspondence should be addressed to Zhi-Gang Wang; wangmath@163.com
}

Received 5 July 2014; Accepted 14 August 2014; Published 28 August 2014

Academic Editor: Rosihan M. Ali

Copyright (C) 2014 Ming-Liang Li et al. This is an open access article distributed under the Creative Commons Attribution License, which permits unrestricted use, distribution, and reproduction in any medium, provided the original work is properly cited.

The main purpose of this paper is to introduce and investigate a certain subclass of meromorphic close-to-convex functions. Such results as coefficient inequalities, convolution property, inclusion relationship, distortion property, and radius of meromorphic convexity are derived.

\section{Introduction and Preliminaries}

Let $\Sigma$ denote the class of functions $f$ of the form

$$
f(z)=\frac{1}{z}+\sum_{n=1}^{\infty} a_{n} z^{n}
$$

which are analytic in the punctured open unit disk

$$
\mathbb{U}^{*}:=\{z: z \in \mathbb{C} 0<|z|<1\}=: \mathbb{U} \backslash\{0\} .
$$

For two functions $f$ and $g$, analytic in $\mathbb{U}$, we say that the function $f$ is subordinate to $g$ in $\mathbb{U}$ and write

$$
f(z) \prec g(z) \quad(z \in \mathbb{U}),
$$

if there exists a Schwarz function $\omega$, which is analytic in $\mathbb{U}$ with

$$
\omega(0)=0, \quad|\omega(z)|<1 \quad(z \in \mathbb{U})
$$

such that

$$
f(z)=g(\omega(z)) \quad(z \in \mathbb{U}) .
$$

Indeed, it is known that

$$
\begin{array}{r}
f(z) \prec g(z) \quad(z \in \mathbb{U}) \Longrightarrow \\
f(0)=g(0), \\
f(\mathbb{U}) \subset g(\mathbb{U}) .
\end{array}
$$

Furthermore, if the function $g$ is univalent in $\mathbb{U}$, then we have the following equivalence:

$$
\begin{array}{r}
f(z) \prec g(z) \quad(z \in \mathbb{U}) \Longleftrightarrow f(0)=g(0), \\
f(\mathbb{U}) \subset g(\mathbb{U}) .
\end{array}
$$

Let $f, g \in \Sigma$, where $f$ is given by (1) and $g$ is defined by

$$
g(z)=\frac{1}{z}+\sum_{n=1}^{\infty} b_{n} z^{n} .
$$

Then the Hadamard product (or convolution) $f * g$ of the functions $f$ and $g$ is defined by

$$
(f * g)(z):=\frac{1}{z}+\sum_{n=1}^{\infty} a_{n} b_{n} z^{n}=:(g * f)(z) .
$$

A function $f \in \Sigma$ is said to be in the class $\mathscr{M} \mathcal{S}^{*}$ of meromorphic starlike functions if it satisfies the inequality

$$
\mathfrak{R}\left(\frac{z f^{\prime}(z)}{f(z)}\right)<0 \quad(z \in \mathbb{U}) .
$$

A function $f \in \Sigma$ is said to be in the class $\mathscr{M} \mathscr{K}$ of meromorphic convex functions if it satisfies the inequality

$$
\mathfrak{R}\left(1+\frac{z f^{\prime \prime}(z)}{f^{\prime}(z)}\right)<0 \quad(z \in \mathbb{U}) .
$$


Moreover, a function $f \in \Sigma$ is said to be in the class $\mathscr{M} \mathscr{C}$ of meromorphic close-to-convex functions if it satisfies the condition

$$
\mathfrak{R}\left(\frac{z f^{\prime}(z)}{g(z)}\right)<0 \quad\left(z \in \mathbb{U} ; g \in \mathscr{M} \mathcal{S}^{*}\right) .
$$

Let

$$
\mathfrak{f}(z)=z+\mathfrak{a}_{2} z^{2}+\mathfrak{a}_{3} z^{3}+\cdots
$$

be analytic in $\mathbb{U}$. If there exists a function $\mathfrak{g} \in \mathscr{K}$ such that

$$
\begin{aligned}
& \left|\frac{\left(z \mathfrak{f}^{\prime}(z) / \mathfrak{g}(z)\right)-1}{\left(z \mathfrak{f}^{\prime}(z) / \mathfrak{g}(z)\right)+(1-2 \alpha)}\right|<\beta \\
& (z \in \mathbb{U} ; 0 \leqq \alpha<1 ; 0<\beta \leqq 1),
\end{aligned}
$$

then we say that $\mathfrak{f} \in \mathscr{C}(\alpha, \beta)$, where $\mathscr{K}$ denotes the usual class of convex functions. The function class $\mathscr{C}(\alpha, \beta)$ was introduced and studied recently by Peng [1] (see also Peng and Han [2], Selvaraj [3], Gao and Zhou [4], Kowalczyk and Leś-Bomba [5], and Xu et al. [6]).

Motivated essentially by the above mentioned function class $\mathscr{C}(\alpha, \beta)$, we now introduce and investigate the following class of meromorphic close-to-convex functions.

Definition 1. A function $f \in \Sigma$ is said to be in the class $\mathscr{M} \mathscr{C}(\alpha, \beta)$ if it satisfies the inequality

$$
\left|\frac{\left(z f^{\prime}(z) / g(z)\right)+1}{\left(z f^{\prime}(z) / g(z)\right)+(2 \alpha-1)}\right|<\beta \quad\left(z \in \mathbb{U} ; g \in \mathscr{M} \mathcal{S}^{*}\right) \text {, }
$$

where (and throughout this paper unless otherwise mentioned) the parameters $\alpha$ and $\beta$ are constrained as follows:

$$
0 \leqq \alpha<1, \quad 0<\beta \leqq 1
$$

It is easy to verify that $f \in \mathscr{M} \mathscr{C}(\alpha, \beta)$ if and only if

$$
-\frac{z f^{\prime}(z)}{g(z)} \prec \frac{1+(1-2 \alpha) \beta z}{1-\beta z} \quad(z \in \mathbb{U}) .
$$

We observe that

$$
\mathfrak{R}\left(\frac{1+(1-2 \alpha) \beta z}{1-\beta z}\right)>0 \quad(z \in \mathbb{U}),
$$

and, thus, the function class $\mathscr{M} \mathscr{C}(\alpha, \beta)$ is a subclass of meromorphic close-to-convex functions.

Clearly, the class $\mathscr{M} \mathscr{C}(\alpha, 1)=: \mathscr{M} \mathscr{C}(\alpha)$ is the familiar class of meromorphic close-to-convex functions of order $\alpha$.

For some recent investigations of meromorphic functions, see, for example, the works of [7-22] and the references cited therein.

To derive our main results, we need the following lemmas.
Lemma 2 (see [23]). Let

$$
f_{1}(z)=1+\sum_{n=1}^{\infty} \mathbf{c}_{n} z^{n}
$$

be analytic in $\mathbb{U}$ and let

$$
f_{2}(z)=1+\sum_{n=1}^{\infty} \mathbf{d}_{n} z^{n}
$$

be analytic and convex in $\mathbb{U}$. If $f_{1} \prec f_{2}$, then

$$
\left|\mathbf{c}_{n}\right| \leqq\left|\mathbf{d}_{1}\right| \quad(n \in \mathbb{N}:=\{1,2, \ldots\}) .
$$

Lemma 3 (see [24]). Suppose that

$$
\mathfrak{h}(z)=\frac{1}{z}+\sum_{n=1}^{\infty} c_{n} z^{n} \in \mathscr{M} \mathcal{S}^{*} .
$$

Then

$$
\left|c_{n}\right| \leqq \frac{2}{n+1} \quad(n \in \mathbb{N}) .
$$

Each of these inequalities is sharp, with the extremal function given by

$$
\mathfrak{h}(z)=z^{-1}\left(1+z^{n+1}\right)^{2 /(n+1)}
$$

Lemma 4 (see [25]). Let $-1 \leqq D<C \leqq 1$ and $-1 \leqq F<E \leqq$ 1. Then,

$$
\frac{1+C z}{1+D z} \prec \frac{1+E z}{1+F z}
$$

if and only if

$$
|E D-F C| \leqq(E-F)-(C-D) .
$$

Lemma 5 (see [26]). Suppose that the function $\mathbf{g} \in \mathscr{M} \mathcal{S}^{*}$. Then

$$
\frac{(1-r)^{2}}{r} \leqq|\mathbf{g}(z)| \leqq \frac{(1+r)^{2}}{r} \quad(|z|=r ; 0<r<1) .
$$

Lemma 6 (see [27]). Suppose that

$$
\mathfrak{p}(z) \prec \frac{1+(1-2 \alpha) z}{1-z} \quad(z \in \mathbb{U} ; 0 \leqq \alpha<1) .
$$

Then,

$$
\begin{aligned}
& \mathfrak{R}\left(\frac{z \mathfrak{p}^{\prime}(z)}{\mathfrak{p}(z)}\right) \\
& \geqq \begin{cases}-\frac{2(1-\alpha) r}{(1+r)[1+(2 \alpha-1) r]} & \left(|z|=r ; 0<r \leqq r_{0}\right), \\
\left(\sqrt{4 \alpha\left(1-r^{2}\right)\left[1+(1-2 \alpha) r^{2}\right]}\right. & \\
\left.-\left[1+(1-2 \alpha) r^{2}\right]\right) & \\
\times\left((1-\alpha)\left(1-r^{2}\right)\right)^{-1} & \left(|z|=r ; r_{0}<r<1\right), \\
-\frac{\alpha}{1-\alpha} & \end{cases}
\end{aligned}
$$


where $r_{0}$ is the unique root of the equation

$$
(2 \alpha-1) r^{4}-2(2 \alpha-1) r^{3}-6 \alpha r^{2}-2 r+1=0
$$

in the interval $(0,1)$. The results are sharp.

In the present paper, we aim at proving some coefficient inequalities, convolution property, inclusion relationship, distortion property, and radius of meromorphic convexity of the class $\mathscr{M} \mathscr{C}(\alpha, \beta)$.

\section{Main Results}

We begin by stating the following coefficient inequality of the class $\mathscr{M} \mathscr{C}(\alpha, \beta)$.

Theorem 7. Suppose that

$$
f(z)=\frac{1}{z}+\sum_{n=1}^{\infty} a_{n} z^{n} \in \mathscr{M} \mathscr{C}(\alpha, \beta) .
$$

Then

$$
\begin{gathered}
\left|a_{1}\right| \leqq 1 \\
\left|a_{n}\right| \leqq \frac{2(1-\alpha) \beta}{n}\left(1+\sum_{k=1}^{n-1} \frac{2}{k+1}\right)+\frac{2}{n(n+1)}
\end{gathered}
$$

$(n \in \mathbb{N} \backslash\{1\})$.

Proof. Let $f \in \mathscr{M} \mathscr{C}(\alpha, \beta)$ and suppose that

$$
p(z):=-\frac{z f^{\prime}(z)}{g(z)} \quad(z \in \mathbb{U}),
$$

where

$$
g(z)=\frac{1}{z}+\sum_{n=1}^{\infty} b_{n} z^{n} \in \mathscr{M} \mathcal{S}^{*}
$$

It follows that

$$
p(z)=1+\sum_{n=1}^{\infty} p_{n} z^{n} \prec \frac{1+(1-2 \alpha) \beta z}{1-\beta z} \quad(z \in \mathbb{U}) .
$$

In view of Lemma 2, we know that

$$
\left|p_{n}\right| \leqq 2(1-\alpha) \beta \quad(n \in \mathbb{N})
$$

By substituting the series expressions of functions $f, g$, and $p$ into (34), we get

$$
\begin{gathered}
\left(1+p_{1} z+p_{2} z^{2}+\cdots+p_{n} z^{n}+p_{n+1} z^{n+1}+\cdots\right) \\
\times\left(\frac{1}{z}+b_{1} z+b_{2} z^{2}+\cdots+b_{n} z^{n}+\cdots\right) \\
=\frac{1}{z}-a_{1} z-2 a_{2} z^{2}-\cdots-n a_{n} z^{n}-\cdots
\end{gathered}
$$

Since $f$ is univalent in $\mathbb{U}^{*}$, it is well known that $\left|a_{1}\right| \leqq 1$.
On the other hand, we find from (38) that

$$
-n a_{n}=b_{n}+p_{1} b_{n-1}+p_{2} b_{n-2}+\cdots+p_{n-1} b_{1}+p_{n+1}
$$

$$
(n \in \mathbb{N} \backslash\{1\}) \text {. }
$$

By noting that $g \in M \mathcal{S}^{*}$, it follows from Lemma 3 that

$$
\left|b_{n}\right| \leqq \frac{2}{n+1} \quad(n \in \mathbb{N}) .
$$

Combining (37), (39), and (40), we have

$$
\begin{array}{r}
n\left|a_{n}\right| \leqq 2(1-\alpha) \beta\left(\frac{2}{n}+\frac{2}{n-1}+\cdots+\frac{2}{3}+1+1\right)+\frac{2}{n+1} \\
(n \in \mathbb{N} \backslash\{1\}) .
\end{array}
$$

Thus, the assertion (33) of Theorem 7 follows directly from (41).

Theorem 8. Let

$$
g(z)=\frac{1}{z}+\sum_{n=1}^{\infty} b_{n} z^{n} \in \mathscr{M} \mathcal{S}^{*} .
$$

If $f \in \Sigma$ satisfies the condition

$$
(1+\beta) \sum_{n=1}^{\infty} n\left|a_{n}\right|+(1+|2 \alpha-1| \beta) \sum_{n=1}^{\infty}\left|b_{n}\right| \leqq 2(1-\alpha) \beta,
$$

then $f \in \mathscr{M} \mathscr{C}(\alpha, \beta)$.

Proof. To prove $f \in \mathscr{M} \mathscr{C}(\alpha, \beta)$, it suffices to show that (15) holds. From (43), we know that

$$
\begin{gathered}
\beta\left(2(1-\alpha)-\sum_{n=1}^{\infty} n\left|a_{n}\right|-|2 \alpha-1| \sum_{n=1}^{\infty}\left|b_{n}\right|\right) \\
\geqq \sum_{n=1}^{\infty} n\left|a_{n}\right|+\sum_{n=1}^{\infty}\left|b_{n}\right|>0 .
\end{gathered}
$$

Now, by the maximum modulus principle, we deduce from (1) and (44) that

$$
\begin{aligned}
& \left|\frac{\left(z f^{\prime}(z) / g(z)\right)+1}{\left(z f^{\prime}(z) / g(z)\right)+(2 \alpha-1)}\right| \\
& \quad=\left|\frac{\sum_{n=1}^{\infty} n a_{n} z^{n+1}+\sum_{n=1}^{\infty} b_{n} z^{n+1}}{\sum_{n=1}^{\infty} n a_{n} z^{n+1}+(2 \alpha-1) \sum_{n=1}^{\infty} b_{n} z^{n+1}+2(\alpha-1)}\right| \\
& <\frac{\sum_{n=1}^{\infty} n\left|a_{n}\right|+\sum_{n=1}^{\infty}\left|b_{n}\right|}{2(1-\alpha)-\sum_{n=1}^{\infty} n\left|a_{n}\right|-|2 \alpha-1| \sum_{n=1}^{\infty}\left|b_{n}\right|} \leqq \beta .
\end{aligned}
$$

This evidently completes the proof of Theorem 8 .

Example 9. By applying Theorem 8, it is obvious to see that the function

$$
f(z)=\frac{1}{z}+z \in \mathscr{M} \mathscr{C}(0,1)
$$


Theorem 10. Let $|\xi|=1$ and $g \in \mathscr{M} \mathcal{S}^{*}$. A function $f \in$ $\mathscr{M} \mathscr{C}(\alpha, \beta)$ if and only if

$$
\begin{array}{r}
f(z) *\left[(1-\beta \xi) \frac{1-2 z}{z(1-z)^{2}}\right] \\
-g(z) *\left\{[1+(1-2 \alpha) \beta \xi] \frac{z^{2}-z+1}{z(1-z)}\right\} \neq 0 \\
\left(z \in \mathbb{U}^{*}\right) .
\end{array}
$$

Proof. A function $f \in \mathscr{M} \mathscr{C}(\alpha, \beta)$ if and only if

$$
-\frac{z f^{\prime}(z)}{g(z)} \neq \frac{1+(1-2 \alpha) \beta \xi}{1-\beta \xi} \quad(z \in \mathbb{U} ;|\xi|=1) .
$$

It is easy to see that condition (48) can be written as

$$
\begin{array}{r}
z f^{\prime}(z)(1-\beta \xi)+g(z)[1+(1-2 \alpha) \beta \xi] \neq 0 \\
\left(z \in \mathbb{U}^{*} ;|\xi|=1\right) .
\end{array}
$$

We observe that

$$
\begin{gathered}
-z f^{\prime}(z)=f(z) *\left(\frac{1}{z}-\frac{z}{(1-z)^{2}}\right)=f(z) * \frac{1-2 z}{z(1-z)^{2}} \\
g(z)=g(z) *\left(\frac{1}{z}+\frac{z}{1-z}\right)=g(z) * \frac{z^{2}-z+1}{z(1-z)} .
\end{gathered}
$$

By substituting (50) into (49), we get the desired assertion (47) of Theorem 10.

Theorem 11. Let

$$
0 \leqq \alpha_{2} \leqq \alpha_{1} \leqq \frac{1}{2}, \quad 0<\beta_{1} \leqq \beta_{2}<1 .
$$

Then,

$$
\mathscr{M} \mathscr{C}\left(\alpha_{1}, \beta_{1}\right) \subset \mathscr{M} \mathscr{C}\left(\alpha_{2}, \beta_{2}\right) .
$$

Proof. Suppose that $f \in \mathscr{M} \mathscr{C}\left(\alpha_{1}, \beta_{1}\right)$. We easily know that

$$
-\frac{z f^{\prime}(z)}{g(z)} \prec \frac{1+\left(1-2 \alpha_{1}\right) \beta_{1} z}{1-\beta_{1} z} \quad(z \in \mathbb{U}) .
$$

By setting $C=1+\left(1-2 \alpha_{1}\right) \beta_{1}, D=-\beta_{1}, E=1+\left(1-2 \alpha_{2}\right) \beta_{2}$, and $F=-\beta_{2}$, it follows from (51) that

$$
\begin{aligned}
\mid E D- & F C \mid \\
= & \left|-\left(1-2 \alpha_{2}\right) \beta_{1} \beta_{2}+\left(1-2 \alpha_{1}\right) \beta_{1} \beta_{2}\right| \\
\leqq & \left|-\left(1-2 \alpha_{2}\right) \beta_{1} \beta_{2}+\left(1-2 \alpha_{1}\right) \beta_{1}^{2}\right| \\
& \quad+\left|-\left(1-2 \alpha_{1}\right) \beta_{1}^{2}+\left(1-2 \alpha_{1}\right) \beta_{1} \beta_{2}\right| \\
\leqq & {\left[\left(1-2 \alpha_{2}\right) \beta_{2}-\left(1-2 \alpha_{1}\right) \beta_{1}\right]+\left(\beta_{2}-\beta_{1}\right) } \\
= & {\left[\left(1-2 \alpha_{2}\right) \beta_{2}-\left(-\beta_{2}\right)\right] } \\
& \quad-\left[\left(1-2 \alpha_{1}\right) \beta_{1}-\left(-\beta_{1}\right)\right] \\
= & (E-F)-(C-D) .
\end{aligned}
$$

In view of Lemma 4 , we deduce that

$$
\begin{array}{r}
-\frac{z f^{\prime}(z)}{g(z)} \prec \frac{1+\left(1-2 \alpha_{1}\right) \beta_{1} z}{1-\beta_{1} z} \prec \frac{1+\left(1-2 \alpha_{2}\right) \beta_{2} z}{1-\beta_{2} z} \\
(z \in \mathbb{U}),
\end{array}
$$

which implies that $f \in \mathscr{M} \mathscr{C}\left(\alpha_{2}, \beta_{2}\right)$. Thus, the assertion (52) of Theorem 11 holds.

Theorem 12. Let $f \in M \mathscr{C}(\alpha, \beta)$. Then,

$$
\begin{aligned}
& \frac{(1-r)^{2}[1-(1-2 \alpha) \beta r]}{r^{2}(1+\beta r)} \\
& \leqq\left|f^{\prime}(z)\right| \leqq \frac{(1+r)^{2}[1+(1-2 \alpha) \beta r]}{r^{2}(1-\beta r)} \\
& (|z|=r ; 0<r<1) .
\end{aligned}
$$

Proof. Let $f \in \mathscr{M} \mathscr{C}(\alpha, \beta)$. By definition, we know that

$$
-\frac{z f^{\prime}(z)}{g(z)} \prec \frac{1+(1-2 \alpha) \beta z}{1-\beta z} \quad(z \in \mathbb{U}) .
$$

Suppose that the function $p$ is defined by (36). Then, we have

$$
\begin{array}{r}
\frac{1-(1-2 \alpha) \beta r}{1+\beta r} \leqq|p(z)| \leqq \frac{1+(1-2 \alpha) \beta r}{1-\beta r}, \\
(|z|=r ; 0<r<1) .
\end{array}
$$

Since $g \in \mathscr{M} \mathcal{S}^{*}$, by Lemma 5, we know that

$$
\frac{(1-r)^{2}}{r} \leqq|g(z)| \leqq \frac{(1+r)^{2}}{r} \quad(|z|=r ; 0<r<1)
$$

Thus, by virtue of (36), (58), and (59), we readily get the assertion (56) of Theorem 12.

Finally, we derive the radius of meromorphic convexity for the class $\mathscr{M} \mathscr{C}(\alpha)$.

Theorem 13. Let $f \in \mathscr{M} \mathscr{C}(\alpha)$ with $0<\alpha<1$. Then,

(1) for $r_{1} \leqq r_{0}$, $f$ is meromorphic convex in $0<|z|<r_{1}$;

(2) for $r_{1}>r_{0}$, $f$ is meromorphic convex in $0<|z|<r_{2}$, where $r_{0}$ is the unique root of the equation

$$
(2 \alpha-1) r^{4}-2(2 \alpha-1) r^{3}-6 \alpha r^{2}-2 r+1=0
$$


in the interval $(0,1)$ and $r_{1}$ and $r_{2}$ are the smallest root of the equations

$$
\begin{gathered}
(2 \alpha-1) r^{3}+3(2 \alpha-1) r^{2}+3 r+1=0, \\
(1-2 \alpha) r^{4}+2(1-2 \alpha) r^{3}+3(1-\alpha) r^{2}+2 r+1=0
\end{gathered}
$$

in the interval $(0,1)$, respectively.

Proof. Let $f \in \mathscr{M} \mathscr{C}(\alpha)$ and suppose that

$$
q(z):=-\frac{z f^{\prime}(z)}{g(z)} \quad(z \in \mathbb{U}) .
$$

Then,

$$
q(z) \prec \frac{1+(1-2 \alpha) z}{1-z} \quad(z \in \mathbb{U})
$$

It follows from (62) that

$$
-z f^{\prime}(z)=q(z) g(z)
$$

Differentiating both sides of (64) logarithmically, we get

$$
-\left(1+\frac{z f^{\prime \prime}(z)}{f^{\prime}(z)}\right)=\frac{z q^{\prime}(z)}{q(z)}+\frac{z g^{\prime}(z)}{g(z)} .
$$

Since $g \in \mathscr{M} \mathcal{S}^{*}$, we know that

$$
\mathfrak{R}\left(\frac{z g^{\prime}(z)}{g(z)}\right) \geqq-\frac{1+r}{1-r} \quad(|z|=r) .
$$

Combining (63), (65), (66), and Lemma 6, we obtain

$$
\begin{aligned}
& -\Re\left(1+\frac{z f^{\prime \prime}(z)}{f^{\prime}(z)}\right) \\
& \geqq\left\{\begin{array}{l}
-\frac{1+r}{1-r} \quad 2(1-\alpha) r \\
\quad-\frac{1}{(1+r)[1+(2 \alpha-1) r]} \quad\left(|z|=r ; 0<r \leqq r_{0}\right), \\
=: \begin{array}{rl}
\widetilde{H}_{\alpha}(r) & \frac{1+r}{1-r} \\
& +\left(\sqrt{4 \alpha\left(1-r^{2}\right)\left[1+(1-2 \alpha) r^{2}\right]}\right. \\
& \left.-\left[1+(1-2 \alpha) r^{2}\right]\right) \\
& \times\left((1-\alpha)\left(1-r^{2}\right)\right)-1 \\
- & \frac{\alpha}{1-\alpha}=: \widetilde{F}_{\alpha}(r)
\end{array} \quad\left(|z|=r ; r_{0}<r<1\right),
\end{array}\right.
\end{aligned}
$$

where $r_{0}$ is the unique root of $(30)$ in the interval $(0,1)$. It follows from (67) that the bound of meromorphic convexity for the class $\mathscr{M} \mathscr{C}(\alpha)$ is determined either by the equation

$$
-\frac{1+r}{1-r}-\frac{2(1-\alpha) r}{(1+r)[1+(2 \alpha-1) r]}=0,
$$

or by the equation

$$
\begin{aligned}
& -\frac{1+r}{1-r} \\
& +\frac{\sqrt{4 \alpha\left(1-r^{2}\right)\left[1+(1-2 \alpha) r^{2}\right]}-\left[1+(1-2 \alpha) r^{2}\right]}{(1-\alpha)\left(1-r^{2}\right)} \\
& -\frac{\alpha}{1-\alpha}=0 .
\end{aligned}
$$

We note that (68) and (69) can be rewritten as follows:

$$
\begin{aligned}
H_{\alpha}(r) & :=(2 \alpha-1) r^{3}+3(2 \alpha-1) r^{2}+3 r+1=0 \\
F_{\alpha}(r) & :=(1-2 \alpha) r^{4}+2(1-2 \alpha) r^{3}+3(1-\alpha) r^{2}+2 r+1 \\
& =0
\end{aligned}
$$

Let $r_{1}$ and $r_{2}$ be the smallest root of the equations $H_{\alpha}(r)=$ 0 and $F_{\alpha}(r)=0$ in the interval $(0,1)$, respectively. By observing that $H_{\alpha}(0)=1>0$, we deduce that $H_{\alpha}(r)>0$ for $r<r_{1}$.

Similarly, we know that $F_{\alpha}(r)>0$ for $r<r_{2}$, since $F_{\alpha}(0)=$ $1>0$.

We observe that

$$
\begin{aligned}
& \widetilde{H}_{\alpha}(r) \geqq 0 \Longleftrightarrow H_{\alpha}(r) \geqq 0, \\
& \widetilde{F}_{\alpha}(r) \geqq 0 \Longleftrightarrow F_{\alpha}(r) \geqq 0 .
\end{aligned}
$$

Thus, when $r_{1} \leqq r_{0}, f$ is meromorphic convex in $0<|z|<r_{1}$; when $r_{1}>r_{0}, f$ is meromorphic convex in $0<|z|<r_{2}$.

The proof of Theorem 13 is thus completed.

\section{Conflict of Interests}

The authors declare that there is no conflict of interests regarding the publication of this paper.

\section{Acknowledgments}

This research was supported by the National Natural Science Foundation under Grant nos. 11301008 and 11226088, the Foundation for Excellent Youth Teachers of Colleges and Universities of Henan Province under Grant no. 2013GGJS146, and the Natural Science Foundation of Educational Committee of Henan Province under Grant no. 14 B110012 of the People's Republic of China. The authors would like to thank the referees for their valuable comments and suggestions which essentially improved the quality of this paper. 


\section{References}

[1] Z.-G. Peng, "On a subclass of close to convex functions," Acta Mathematica Scientia B, vol. 30, no. 5, pp. 1449-1456, 2010.

[2] Z.-G. Peng and Q.-Q. Han, "On the coefficients of several classes of bi-univalent functions," Acta Mathematica Scientia B, vol. 34, no. 1, pp. 228-240, 2014.

[3] C. Selvaraj, "A subclass of close-to-convex functions," Southeast Asian Bulletin of Mathematics, vol. 28, no. 1, pp. 113-123, 2004.

[4] C.-Y. Gao and S.-Q. Zhou, "On a class of analytic functions related to the starlike functions," Kyungpook Mathematical Journal, vol. 45, no. 1, pp. 123-130, 2005.

[5] J. Kowalczyk and E. Leś-Bomba, "On a subclass of close-toconvex functions," Applied Mathematics Letters, vol. 23, no. 10, pp. 1147-1151, 2010.

[6] N. Xu, D.-G. Yang, and S. Owa, "On strongly starlike multivalent functions of order $\beta$ and type $\alpha$," Mathematische Nachrichten, vol. 283, no. 8, pp. 1207-1218, 2010.

[7] R. M. Ali and V. Ravichandran, "Classes of meromorphic $\alpha$ convex functions," Taiwanese Journal of Mathematics, vol. 14, no. 4, pp. 1479-1490, 2010.

[8] R. M. Ali, V. Ravichandran, and N. Seenivasagan, "Subordination and superordination of the Liu-Srivastava linear operator on meromorphic functions," Bulletin of the Malaysian Mathematical Sciences Society, vol. 31, no. 2, pp. 193-207, 2008.

[9] R. M. Ali, V. Ravichandran, and N. Seenivasagan, "On subordination and superordination of the multiplier transformation for meromorphic functions," Bulletin of the Malaysian Mathematical Sciences Society, vol. 33, no. 2, pp. 311-324, 2010.

[10] J. Dziok, "Classes of meromorphic functions associated with conic regions," Acta Mathematica Scientia B, vol. 32, no. 2, pp. 765-774, 2012.

[11] J. Dziok, G. Murugusundaramoorthy, and J. Sokół, "On certain class of meromorphic functions with positive coefficients," Acta Mathematica Scientia B, vol. 32, no. 4, pp. 1376-1390, 2012.

[12] M. Nunokawa and O. P. Ahuja, "On meromorphic starlike and convex functions," Indian Journal of Pure and Applied Mathematics, vol. 32, no. 7, pp. 1027-1032, 2001.

[13] V. Ravichandran, S. Sivaprasad Kumar, and K. G. Subramanian, "Convolution conditions for spirallikeness and convex spirallikeness of certain meromorphic $p$-valent functions," Journal of Inequalities in Pure and Applied Mathematics, vol. 5, no. 1, article 11, pp. 1-7, 2004.

[14] L. Shi, J.-P. Yi, and Z.-G. Wang, "On certain subclass of meromorphic close-to-convex functions," Applied Mathematics and Computation, vol. 219, no. 20, pp. 10143-10149, 2013.

[15] H. M. Srivastava, D.-G. Yang, and N. Xu, "Some subclasses of meromorphically multivalent functions associated with a linear operator," Applied Mathematics and Computation, vol. 195, no. 1, pp. 11-23, 2008.

[16] Z.-G. Wang, Z.-H. Liu, and A. Cătaş, "On neighborhoods and partial sums of certain meromorphic multivalent functions," Applied Mathematics Letters, vol. 24, no. 6, pp. 864-868, 2011.

[17] Z.-G. Wang, Z.-H. Liu, and R.-G. Xiang, "Some criteria for meromorphic multivalent starlike functions," Applied Mathematics and Computation, vol. 218, no. 3, pp. 1107-1111, 2011.

[18] Z. Wang, Y. Sun, and N. Xu, "Some properties of certain meromorphic close-to-convex functions," Applied Mathematics Letters, vol. 25, no. 3, pp. 454-460, 2012.

[19] Z.-G. Wang, H. M. Srivastava, and S.-M. Yuan, "Some basic properties of certain subclasses of meromorphically starlike functions," Journal of Inequalities and Applications, vol. 2014, article 29, 2014.

[20] Z.-G. Wang, Y. Sun, and Z.-H. Zhang, "Certain classes of meromorphic multivalent functions," Computers \& Mathematics with Applications, vol. 58, no. 7, pp. 1408-1417, 2009.

[21] Y.-H. Xu, B. A. Frasin, and J.-L. Liu, "Certain sufficient conditions for starlikeness and convexity of meromorphically multivalent functions," Acta Mathematica Scientia B, vol. 33, no. 5, pp. 1300-1304, 2013.

[22] S. Yuan, Z. Liu, and H. M. Srivastava, "Some inclusion relationships and integral-preserving properties of certain subclasses of meromorphic functions associated with a family of integral operators," Journal of Mathematical Analysis and Applications, vol. 337, no. 1, pp. 505-515, 2008.

[23] A. W. Goodman, Univalent Functions, vol. 1, Polygonal Publishing House, Washington, NJ, USA, 1983.

[24] J. Clunie, "On meromorphic schlicht functions," Journal of the London Mathematical Society, vol. 34, pp. 215-216, 1959.

[25] H. Silverman and E. M. Silvia, "Subclasses of starlike functions subordinate to convex functions," Canadian Journal of Mathematics, vol. 27, pp. 48-61, 1985.

[26] C. Pommerenke, "On meromorphic starlike functions," Pacific Journal of Mathematics, vol. 13, pp. 221-235, 1963.

[27] W. Janowski, "Some extremal problems for certain families of analytic functions," Annales Polonici Mathematici, vol. 28, pp. 297-326, 1973. 


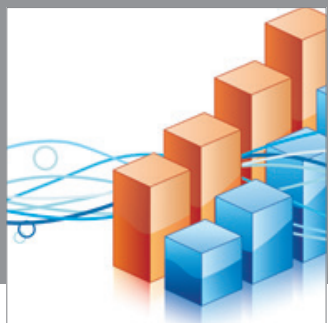

Advances in

Operations Research

mansans

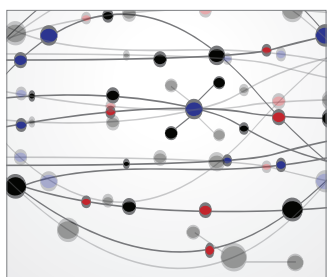

The Scientific World Journal
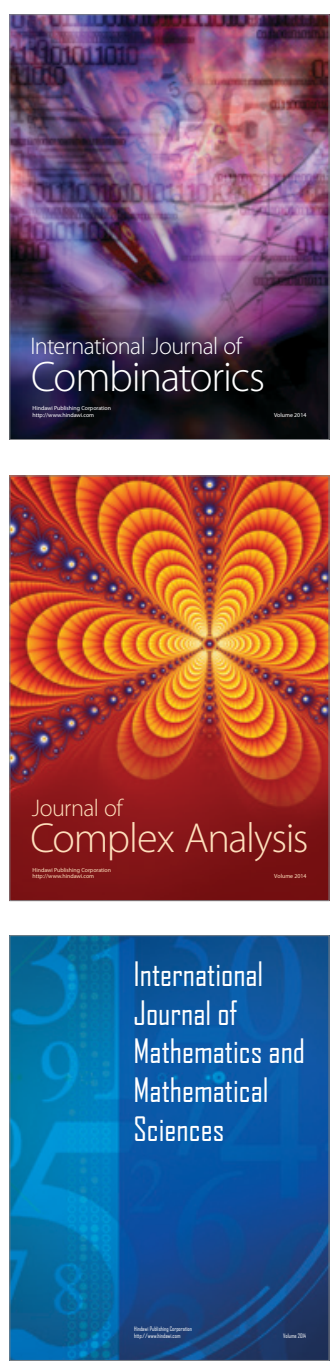
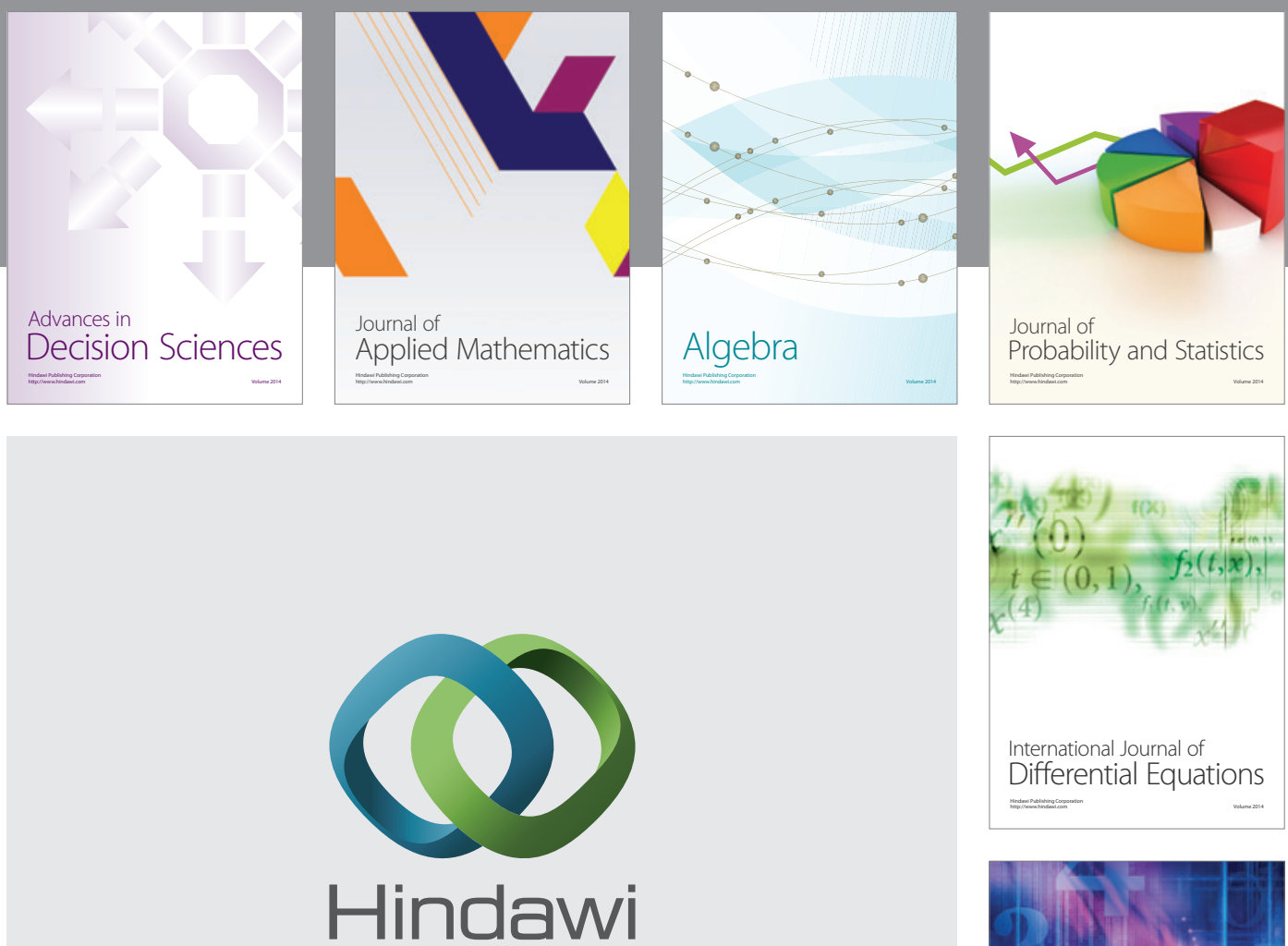

Submit your manuscripts at http://www.hindawi.com
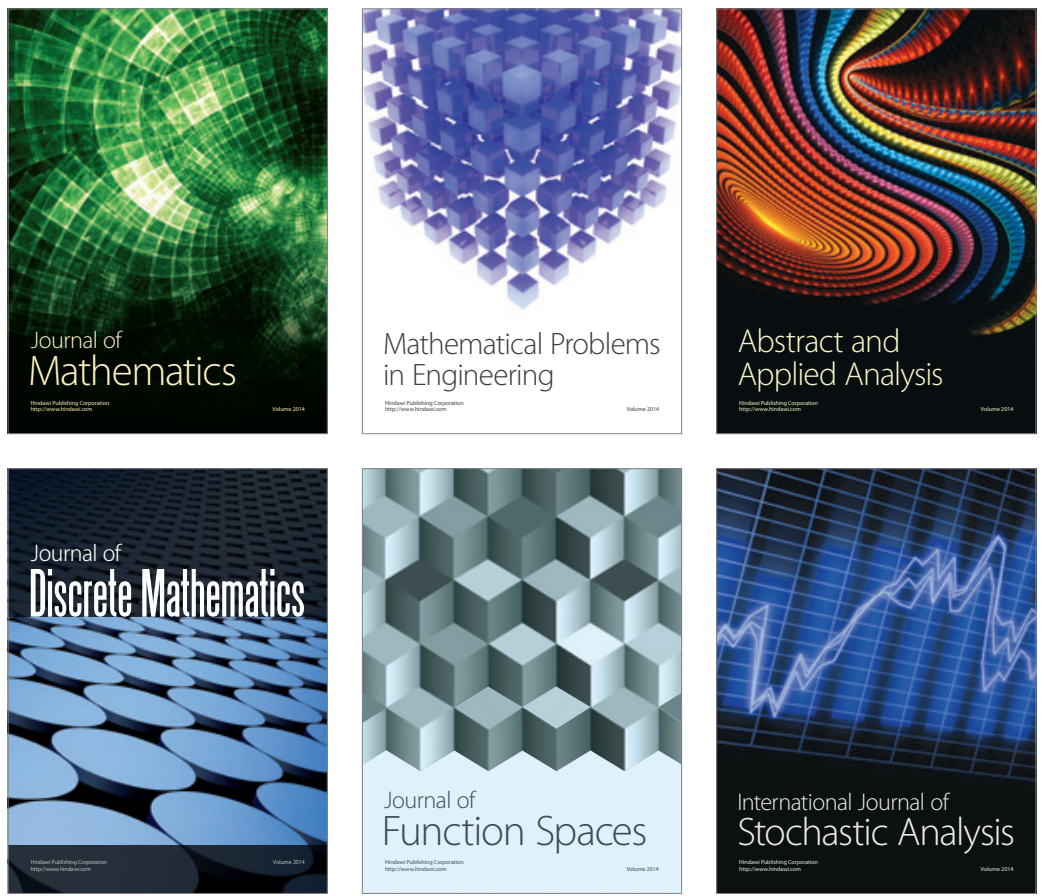

Journal of

Function Spaces

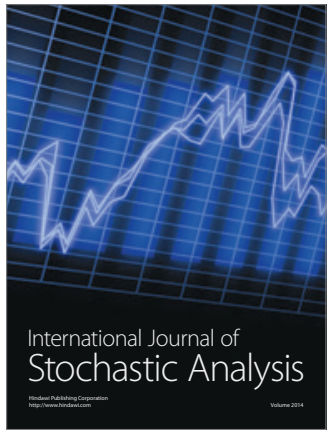

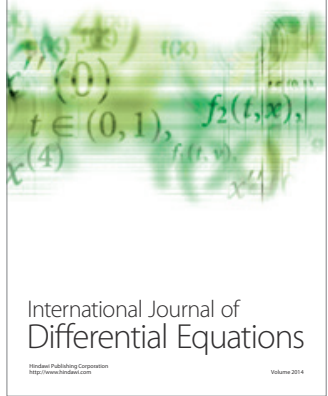
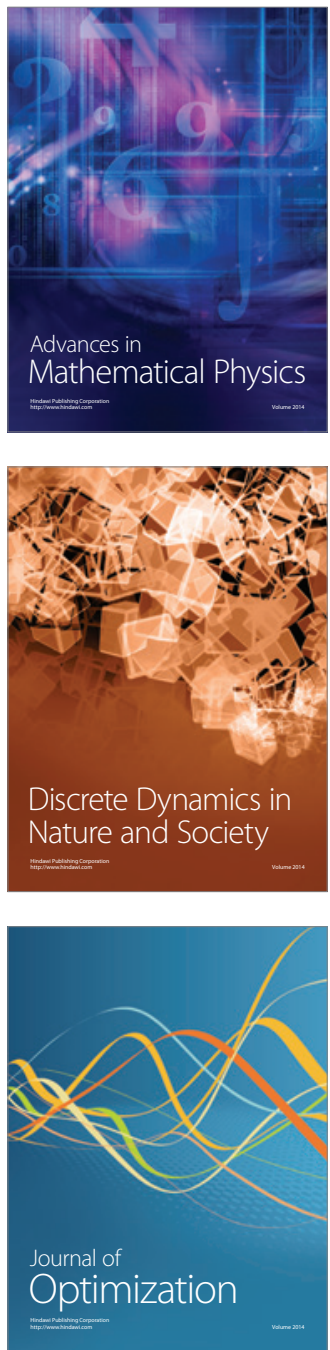